\title{
Post COVID-19 persistent fatigue: a proposal for rehabilitative interventions in the spa setting
}

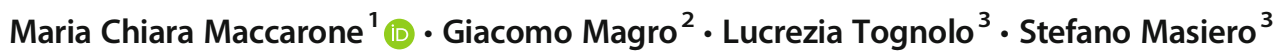

Received: 9 March 2021 / Revised: 19 May 2021 / Accepted: 31 May 2021 / Published online: 4 June 2021

(C) ISB 2021

\begin{abstract}
With this letter to Editor, we aim at offering our viewpoint on the potential use of the spa resources to contribute to the treatment of persistent COVID-19 sequelae. In particular, our argumentations focus on the comprehensive management of fatigue persisting after the resolution of the acute infection. This sequela seems to be the most frequent disabling outcome, leading to a delay in social reintegration and return to working life.

We suggest that spa facilities including multidisciplinary interventions and trained staffs could be an appropriate setting for providing rehabilitative protocols to treat chronic fatigue in post COVID-19 subjects. Spa treatments may improve the physical symptoms of chronic fatigue and modulate the immune imbalance, reducing persisting inflammation. Moreover, in the spa setting, specific respiratory rehabilitation and neuropsychological interventions could be provided. The rehabilitative protocol could also properly address comorbidities.

In conclusion, respecting the hygiene and preventive measures, the spa setting could represent the appropriate environment to take care of post-COVID chronic fatigue, offering adequate and multidisciplinary rehabilitation protocols. Further, prospective trials on this topic are needed to fully evaluate the positive impact of a comprehensive rehabilitative treatment in the spa environment for subjects with post-COVID-19 fatigue.
\end{abstract}

\section{Dear Editor,}

About a year after the outbreak of the COVID-19 pandemic, it has now been settled that the long-term outcomes of this infection can be serious and persistent. After recovery from the infection many subjects do not return to full health, despite being medically deemed to be recovered from their primary illness, and may not resume work (Townsend et al. 2020) due to the persistent and highly disabling outcomes.

Among the disabling prolonged sequelae of acute COVID19 , the most frequent one is persistent fatigue (53.1-72\%) (Amenta et al. 2020; Carfi et al. 2020; Halpin et al. 2020). Chronic fatigue can occur in patients discharged from

Maria Chiara Maccarone

mariachiara.maccarone93@gmail.com

1 Physical Medicine and Rehabilitation School, University of Padova, Via Giustiniani 3, 35128 Padua, Italy

2 Physical Medicine and Rehabilitation School, University of Padova, Padua, Italy

3 Rehabilitation Unit, Department of Neuroscience, University of Padova, Padua, Italy
Intensive Care Unit (ICU) or, although less frequently, in subjects recovered from mild infection (Amenta et al. 2020). Recent evidence has shown that chronic post-COVID syndrome lasts beyond 12 weeks and requires a multidisciplinary and integrated rehabilitation approach, based on local needs and resources, with staff expertise in treating fatigue and respiratory symptoms (Shah et al. 2021).

Since patients with chronic fatigue are increasing worldwide in proportion to the increase in COVID-19 infection cases; there is an urgent need for innovative and effective protocols to treat these subjects. However, to the best of our knowledge, to date there are no established treatments to counteract the disabling outcomes of post-COVID-19 syndrome and persisting fatigue. We believe that spa facilities, offering multidisciplinary interventions and professional staffs, may be an appropriate setting to provide rehabilitative protocols to treat chronic fatigue in post COVID-19 subjects. This will reduce the burden, in both disabling and economic terms, of COVID-19 infection.

Although the pathophysiology of post-COVID fatigue syndrome is not yet clarified, it has been hypothesized that, similar to what occurred in SARS-CoV, persistent sequelae (Moldofsky and Patcai 2011), the virus-induced "cytokine 
storm" could be responsible for the development of chronic fatigue (Islam et al. 2020; Amenta et al. 2020). Possibly, the build-up of pro-inflammatory cytokines in the Central Nervous System may contribute to the symptoms as they pass through the blood brain barrier in circumventricular organs, leading to autonomic disorder among which is unremitting fatigue (Perrin et al. 2020). In particular, overexpression of interleukin 6 (IL-6) would be linked to persistent inflammation and fatigue (Perrin et al. 2020). Immersions in mineralrich waters and mud baths have been demonstrated to be effective in reducing pro-inflammatory cytokines, including IL6 levels (Galvez et al. 2020; Maccarone et al. 2020; Masiero et al. 2020a), and their employment could contrast fatiguerelated cytokines accumulation. Moreover, spa applications could stimulate the release of anti-inflammatory cytokines, restoring the immune imbalance (Maccarone et al. 2020; Masiero et al. 2020a).

Spa interventions could also act on the physical symptoms associated to chronic fatigue, such as post-exertional malaise, muscle pain, and polyarthralgia (Yancey and Thomas 2012). Aerobic exercise and strength training in mineral-rich water can contribute to improve muscles function and to reduce muscular pain in post-COVID-19 subjects (Masiero et al. 2020b).

A randomized multicenter trial demonstrated that a spa therapy protocol including hydromassage baths, mud applications, body jet showers, water affusion massages, and collective exercise in a mineral water pool could significantly improve fatigue in patients with fibromyalgia (Maindet et al. 2021).

There is no doubt that pulmonary pathology contributes to fatigue (Halpin et al. 2021). In the spa setting, a specific respiratory rehabilitation service could be provided, through the combination of mineral-rich water inhalations, respiratory exercise and aerobic training (Masiero et al. 2020b).

In addition, since fatigue is frequently related to neurological symptoms, like unrefreshing sleep, impaired memory, lack of concentration, and headaches (Yancey and Thomas 2012), its management in the spa setting can also be appropriate to treat these neuropsychological outcomes. Indeed, in the spa setting, patients could receive not only a tailored program for physical and respiratory rehabilitation, but also neuropsychological interventions in a mind-supportive environment (Antonelli and Donelli 2020; Masiero et al. 2020b). Actually, interventions in the spa setting have been shown to be effective in reducing fatigue having a positive effect on mood, pain, and cognitive function, through the reduction of stress, the increase of stress resilience, and the changes in cortisol levels (Rapolien et al. 2016; Yang et al. 2018; Dilekçi et al. 2020; Antonelli and Donelli 2018).

Finally, in the thermal environment, it is also possible to take care of comorbidities, such as advanced age, obesity, and rheumatic diseases, which may play a role in the initiation and development of the chronic inflammatory substrate (Bektas et al. 2018; Wueest and Konrad 2020).

The exploitation of spa facilities may represent a valid outof-hospital alternative to the traditional rehabilitation setting, allowing to treat all the aspects of the complex post-COVID fatigue and to reduce the pressure on hospital rehabilitation units, which are overworked by acute rehabilitation of COVID-19 patients (Maccarone and Masiero 2021). Providing an out-of-hospital rehabilitation setting may also aid patients emerging from long hospitalization: distancing from the hospital may contribute to further improve mental wellbeing in post-COVID-19 subjects.

The rehabilitative program in thermal environment should last at least two weeks with 12 complete treatments, in conformity with the classical duration of thermal treatments. In case of need, the treatment period should be modified according to the evolution of symptoms.

We would like to remark that post-COVID-19 subjects accessing spa treatments must not be infectious anymore (two consecutive negative RT-PCR results and fever resolution) (Antonelli and Donelli 2020; Masiero et al. 2020b; Centers for Disease Control and Prevention 2020a). The Centers for Disease Control and Prevention Concerning stated that there is no evidence that COVID-19 can spread to people through the water used in pools, hot tubs, and spas and a proper disinfection should ensure the inactivation of the SARS-CoV-2 virus (Centers for Disease Control and Prevention 2020b). Access to rehabilitation protocols in the spa environment should be allowed if the requisites of interpersonal distancing, recirculation of the air, and surgical mask wearing are guaranteed, and adequate and frequent sanitization interventions are performed. To ensure distancing, the maximum number of subjects present at the same time in each room should be defined and an access control system that also verifies body temperature should be performed (Clementi et al. 2020).

The development of a multidisciplinary rehabilitation service in the spa setting should offer the appropriate treatment for chronic fatigue, avoiding it to become established with persistent disabilities, and will allow to monitor patients, contributing to expand current scientific knowledge.

Even if the theoretical arguments are sufficient to consider this rehabilitative approach, a thorough scientific assessment should be performed during the applications of the spa protocols. Prospective clinical studies in the future will be able to verify the efficacy and cost effectiveness of protocols in the spa environment for patients with long-term post-COVID outcomes.

Supplementary Information The online version contains supplementary material available at https://doi.org/10.1007/s00484-021-02158-1. 


\section{References}

Amenta EM, Spallone A, Rodriguez-Barradas MC, El Sahly HM, Atmar RL, Kulkarni PA (2020) Postacute COVID-19: an overview and approach to classification. Open Forum Infect Dis 7(12):ofaa509. https://doi.org/10.1093/ofid/ofaa509

Antonelli M, Donelli D (2018) Effects of balneotherapy and spa therapy on levels of cortisol as a stress biomarker: a systematic review. Int $\mathrm{J}$ Biometeorol 62:913-924. https://doi.org/10.1007/s00484-018$1504-8$

Antonelli M, Donelli D (2020) Respiratory rehabilitation for postCOVID19 patients in spa centers: first steps from theory to practice. Int J Biometeorol 64(10):1811-1813. https://doi.org/10.1007/ s00484-020-01962-5

Bektas A, Schurman SH, Sen R, Ferrucci L (2018) Aging, inflammation and the environment. Exp Gerontol 105:10-18. https://doi.org/10. 1016/j.exger.2017.12.015

Carfi A, Bernabei R, Landi F (2020) Persistent symptoms in patients after acute COVID-19. JAMA 324(6):603-605

Clementi M, Signorelli C, Romano Spica V, Vitali M, Conti M, Vitale M (2020) Protocols and self-checking plans for the safety of postCOVID-19 balneotherapy. Acta Biomed 91(9-S):40-49. https:// doi.org/10.23750/abm.v91i9-S.10167

Centers for Disease Control and Prevention (2020a) Discontinuation of transmission-based precautions and disposition of patients with COVID-19 in healthcare settings (Interim Guidance). https://www. cdc.gov/coronavirus/2019-ncov/hcp/disposition-hospitalizedpatients.html. Accessed 31 May 2021

Centers for Disease Control and Prevention (2020b) Water and COVID19 FAQs information about drinking water, treated recreational water, and wastewater. https://www.cdc.gov/coronavirus/2019-ncov/ php/water.html. Accessed 31 May 2021

Dilekçi E, Özkuk K, Kaki B (2020) The short-term effects of balneotherapy on pain, disability and fatigue in patients with chronic low back pain treated with physical therapy: a randomized controlled trial. Complement Ther Med 54:102550. https://doi.org/10. 1016/j.ctim.2020.102550

Galvez I, Torres-Piles S, Ortega E (2020) Effect of mud-bath therapy on the innate/inflammatory responses in elderly patients with osteoarthritis: a discussion of recent results and a pilot study on the role of the innate function of monocytes. Int J Biometeorol 64(6):927-935. https://doi.org/10.1007/s00484-019-01748-4

Halpin SJ, McIvor C, Whyatt G, Adams A, Harvey O, McLean L, Walshaw C, Kemp S, Corrado J, Singh R, Collins T, O'Connor RJ, Sivan M (2020) Postdischarge symptoms and rehabilitation needs in survivors of COVID-19 infection: a cross-sectional evaluation. J Med Virol 93:1013-1022. https://doi.org/10.1002/jmv. 26368

Halpin S, O'Connor R, Sivan M (2021) Long COVID and chronic COVID syndromes. J Med Virol 93:1242-1243. https://doi.org/10. 1002/jmv.26587

Islam MF, Cotler J, Jason LA (2020) Post-viral fatigue and COVID-19: lessons from past epidemics. Fatigue: Biomedicine, Health \& Behavior 8(2):61-69. https://doi.org/10.1080/21641846.2020. 1778227

Maccarone MC, Magro G, Solimene U, Masiero S (2020) The effects of balneotherapy on human immune function: should baths and mud applications have a role during Covid-19 pandemic? Bull Rehab Med 97(3):22-24. https://doi.org/10.38025/2078-1962-2020-97-322-24

Maccarone MC, Masiero S (2021) The important impact of COVID-19 pandemic on the reorganization of a Rehabilitation Unit in a National Healthcare System hospital in Italy: lessons from our experience [published online ahead of print, 2021 Jan 25]. Am J Phys Med Rehabil 100:327-330. https://doi.org/10.1097/PHM. 0000000000001707

Maindet C, Maire A, Vermorel C, Cracowski C, Rolland C, Forestier R, Comte A, Roques CF, Serra E, Bosson JL (2021) Spa therapy for the treatment of fibromyalgia: an open, randomized multicenter trial. J Pain S1526-5900(21):00031-00036. https://doi.org/10.1016/j.jpain. 2021.02 .010

Masiero S, Maccarone MC, Magro G (2020a) Balneotherapy and human immune function in the era of COVID-19. Int J Biometeorol 64(8): 1433-1434. https://doi.org/10.1007/s00484-020-01914-z

Masiero S, Maccarone MC, Agostini F (2020b) Health resort medicine can be a suitable setting to recover disabilities in patients tested negative for COVID-19 discharged from hospital? A challenge for the future. Int J Biometeorol 64:1807-1809. https://doi.org/10.1007/ s00484-020-01947-4

Moldofsky H, Patcai J (2011) Chronic widespread musculoskeletal pain, fatigue, depression and disordered sleep in chronic post-SARS syndrome; a case-controlled study. BMC Neurol 11:37. https://doi.org/ 10.1186/1471-2377-11-37

Perrin R, Riste L, Hann M, Walther A, Mukherjee A, Heald A (2020) Into the looking glass: post-viral syndrome post COVID-19. Med Hypotheses 144:110055 j.mehy.2020.110055

Rapolien L, Razbadauskas A, Slyga J, Martinknas A (2016) Stress and fatigue management using balneotherapy in a short-time randomized controlled trial. Evid Based Complement Alternat Med 2016: 9631684-9631610. https://doi.org/10.1155/2016/9631684

Shah W, Hillman T, Playford ED, Hishmeh L (2021) Managing the long term effects of covid-19: summary of NICE, SIGN, and RCGP rapid guideline. BMJ 372:n136

Townsend L, Dyer AH, Jones K, Dunne J, Mooney A, Gaffney F, O'Connor L, Leavy D, O'Brien K, Dowds J, Sugrue JA, Hopkins D, Martin-Loeches I, Ni Cheallaigh C, Nadarajan P, McLaughlin AM, Bourke NM, Bergin C, O'Farrelly C, Bannan C, Conlon N (2020) Persistent fatigue following SARS-CoV-2 infection is common and independent of severity of initial infection. PLoS One 15(11):e0240784. https://doi.org/10.1371/journal.pone.0240784

Wueest S, Konrad D (2020) The controversial role of IL-6 in adipose tissue on obesity-induced dysregulation of glucose metabolism. Am J Physiol Endocrinol Metab 319(3):E607-E613. https://doi.org/10. 1152/ajpendo.00306.2020

Yancey JR, Thomas SM (2012) Chronic fatigue syndrome: diagnosis and treatment. Am Fam Physician 86(8):741-746

Yang B, Qin QZ, Han LL, Lin J, Chen Y (2018) Spa therapy (balneotherapy) relieves mental stress, sleep disorder, and general health problems in sub-healthy people. Int J Biometeorol 62(2):261272. https://doi.org/10.1007/s00484-017-1447-5

Publisher's note Springer Nature remains neutral with regard to jurisdictional claims in published maps and institutional affiliations. 